Nonlin. Processes Geophys., 20, 841-856, 2013

www.nonlin-processes-geophys.net/20/841/2013/

doi:10.5194/npg-20-841-2013

(c) Author(s) 2013. CC Attribution 3.0 License.

(c) (i)

\title{
Momentum and buoyancy transfer in atmospheric turbulent boundary layer over wavy water surface - Part 2: Wind-wave spectra
}

\author{
Yu. I. Troitskaya ${ }^{1,4}$, E. V. Ezhova ${ }^{1,4}$, D. A. Sergeev ${ }^{1,4}$, A. A. Kandaurov $^{1,4}$, G. A. Baidakov ${ }^{1}$, M. I. Vdovin ${ }^{1,4}$, and \\ S. S. Zilitinkevich ${ }^{2,3,4,5,6}$ \\ ${ }^{1}$ Institute of Applied Physics RAS, Nizhniy Novgorod, Nizhniy Novgorod, Russia \\ ${ }^{2}$ Finnish Meteorological Institute, Helsinki, Finland \\ ${ }^{3}$ Department of Physics, University of Helsinki, Finland \\ ${ }^{4}$ Department of Radiophysics, N.I. Lobachevski State University of Nizhniy Novgorod, Russia \\ ${ }^{5}$ Institute of Geography RAS, Moscow, Russia \\ ${ }^{6}$ Nansen Environmental and Remote Sensing Centre, Bergen, Norway \\ Correspondence to: Yu. I. Troitskaya (yuliya@hydro.appl.sci-nnov.ru)
}

Received: 28 March 2013 - Revised: 26 July 2013 - Accepted: 30 August 2013 - Published: 29 October 2013

\begin{abstract}
Drag and mass exchange coefficients are calculated within a self-consistent problem for the wave-induced air perturbations and mean velocity and density fields using a quasi-linear model based on the Reynolds equations with down-gradient turbulence closure. This second part of the report is devoted to specification of the model elements: turbulent transfer coefficients and wave numberfrequency spectra. It is shown that the theory agrees with laboratory and field experimental data well when turbulent mass and momentum transfer coefficients do not depend on the wave parameters. Among several model spectra better agreement of the theoretically calculated drag coefficients with TOGA (Tropical Ocean Global Atmosphere) COARE (Coupled Ocean-Atmosphere Response Experiment) data is achieved for the Hwang spectrum (Hwang, 2005) with the high frequency part completed by the Romeiser spectrum (Romeiser et al., 1997).
\end{abstract}

\section{Introduction}

The most important characteristics that determine interaction between atmosphere and ocean are fluxes of momentum, heat and moisture. In numerical weather and climate models they are parameterized through the dimensionless exchange coefficients (like surface drag coefficient $C_{\mathrm{D}}$, sensible heat transfer coefficient or the Stanton number $C_{h}$ and moisture exchange coefficient or the Dalton number $C_{\mathrm{e}}$ ). Note that heat and moisture transfer coefficients are usually considered equal (see, e.g., Fairall et al., 2003). Thus, it is possible to describe the effects of marine atmospheric boundary layer (hereafter referred to as MABL) stratification using one equation for the air density allowing for both heat and moisture contributions. Below we will use the mass exchange coefficient $C_{\rho}=C_{h}=C_{\mathrm{e}}$ for parameterization of the turbulent mass flux due to heat and moisture fluxes. Dependencies of the drag coefficient on wind speed and wave parameters were investigated in numerous field and laboratory experiments and a number of theoretical and numerical models of the wind-wave coupling were developed (see, e.g., Makin et al., 1995 and references therein). It was shown that $C_{\mathrm{D}}$ increases with wind speed due to increase of the form drag of surface waves, caused by broadening of their spectrum. For the mass transfer coefficient $C_{\rho}$ the similar dependencies are much less studied. In particular, a role of the wave disturbances induced in the airflow by the surface waves in mass transfer is not quite understood. Observations and laboratory data show that the dependence of $C_{\rho}$ on wind speed is weaker than for $C_{\mathrm{D}}$, and there are differences in the character of the dependence in different data sets. For example, the algorithm COARE 3.0 (Fairall et al., 2003) indicates a slight increase in $C_{\rho}$ with increasing wind speed $U_{10}$. A similar dependence 
was reported by Brut et al. (2005) and in the laboratory experiments by Ocampo-Torres et al. (1994), but the dependence of $C_{\rho}$ on the wind speed was not found by Drennan et al. (2007). The weak dependence of the $C_{\rho}$ on $U_{10}$ was confirmed in the theoretical models by Makin and Mastenbroek (1996), but the character of the dependence (increasing or decreasing) was sensitive to the choice of model.

This is the second paper of a two-part report aimed at investigating the effect of surface waves on the mass exchange in MABL. The formulation of the problem is very similar to the approach by Makin and Mastenbroek (1996) and the main difference is in the model of MABL used. Principle equations of the model are presented in the first part (Troitskaya et al., 2013), hereafter referred to as Part 1. It is a self-consistent quasi-linear model of MABL based on the Reynolds equations with down-gradient turbulence closure, which extents to the case of a stratified MABL, the model developed for the case of homogeneous atmosphere by Reutov and Troitskaya (1996) and Troitskaya and Rybushkina (2008). The expressions for the turbulent transfer coefficients are selected based on comparison with available experimental data and the results of the special experiments carried out in a wind-wave flume to investigate velocity and temperature distribution in the stratified air boundary layer above the water surface disturbed by paddle-generated waves. Description of the wind-wave coupling within the model is based on the solution of the coupled equations for the disturbances induced in the airflow by the surface waves and the equations for the mean wind flow velocity and temperature. Within quasi-linear approximation, the waveinduced disturbances in the airflow are described in linear approximation. The only nonlinear effect taken into account is the wave momentum transfer from wind to waves. The quasilinear model for neutrally stratified MABL was recently verified by comparison with experimental results by Troitskaya et al. (2011) and direct numerical simulation by Druzhinin et al. (2012).

Parameters of the surface waves are an important input to the model. In the quasi-linear approximation used, wave number-frequency spectrum is sufficient for calculations of momentum and mass fluxes. In the first part of this report (Part 1), we considered an idealized case of a single harmonic wave at the water surface propagating in the same direction as wind. In this paper we examine waves with continuous two-dimensional wave number spectra. Sensitivity of the calculated exchange coefficients $C_{\mathrm{D}}$ and $C_{\rho}$ to the wind wave spectrum used in the model is tested. Several model spectra are considered, including Elfouhaily et al. (1997), Apel (1994), Romeiser et al. (1997), Hwang et al. (1996), Hwang (1997, 2005), Hwang et al. (2001a, b), and Hwang and Wang (2004). Calculated values of exchange coefficients are compared to the data collected in the COARE 3.0 algorithm (Fairall et al., 2003).

The structure of the paper is as follows. The modification of the basic equations of a quasi-linear model of a turbu- lent stratified boundary layer above a wavy water surface to the case of the two-dimensional wave spectra are presented in Sect. 2. Section 3 contains short descriptions of wind wave spectra used in the model. An appropriate model of the turbulent transfer coefficient is discussed in Sect. 4, based on comparison with the TOGA (Tropical Ocean Global Atmosphere) COARE (Coupled Ocean-Atmosphere Response Experiment) experimental data and data from the wind-wave flume experiments. Sensitivity of the model to the wind wave spectrum is discussed in Sect. 5.

\section{Basic equations of a quasi-linear model of a turbulent stratified boundary layer above a wavy water surface}

The description of the air turbulent boundary layer can be extended to the case of the random water waves by the same approach as in Troitskaya and Rybushkina (2008). In this case a random surface elevation field is presented as a FourierStieltjes integral:

$\zeta(\boldsymbol{r}, t)=\int \mathrm{d} A(\boldsymbol{k}, \omega) e^{i(\boldsymbol{k} \boldsymbol{r}-\omega t)}$,

where $\boldsymbol{k}=\left(k_{x}, k_{y}\right)$ is a two-dimensional wave vector.

In addition, for a statistically homogeneous and stationary surface elevation field, the following expression holds:

$$
\begin{aligned}
& \left\langle\mathrm{d} A(\boldsymbol{k}, \omega) \mathrm{d} A\left(\boldsymbol{k}_{1}, \omega_{1}\right)\right\rangle= \\
& \quad F(\boldsymbol{k}, \omega) \delta\left(\boldsymbol{k}-\boldsymbol{k}_{1}\right) \delta\left(\omega-\omega_{1}\right) \mathrm{d} \boldsymbol{k} \mathrm{d} \boldsymbol{k}_{1} \mathrm{~d} \omega \mathrm{d} \omega_{1},
\end{aligned}
$$

where $F(\boldsymbol{k}, \omega)$ is a wave number-frequency elevation spectrum.

As in the case of a single harmonic wave, in order to avoid geometric nonlinearity, we introduce the curvilinear coordinates $\left(\zeta_{1}, \zeta_{2}, \eta\right)$ with transformation to the Cartesian coordinates $(x, y, z)$ given by the following formulas:

$$
\begin{aligned}
& x=\zeta_{1}+\int i \cos \theta e^{i\left(k\left(\zeta_{1} \cos \theta+\zeta_{2} \sin \theta\right)-\omega t\right)-k \eta-i \phi} \mathrm{d} A, \\
& y=\zeta_{2}+\int i \sin \theta e^{i\left(k\left(\zeta_{1} \cos \theta+\zeta_{2} \sin \theta\right)-\omega t\right)-i \phi-k \eta} \mathrm{d} A, \\
& z=\eta+\int e^{i\left(k\left(\zeta_{1} \cos \theta+\zeta_{2} \sin \theta\right)-\omega t\right)-i \phi-k \eta} \mathrm{d} A .
\end{aligned}
$$

Here $\theta$ is an angle from the wind direction. In the linear approximation, coordinate surface $\eta=0$ coincides with a wavy water surface.

Similarly to Troitskaya and Rybushkina (2008), windwave interaction is considered in the quasi-linear approximation; thus, different harmonics are independent. Next, one can introduce a curvilinear reference frame for each harmonic, following this particular wave. By means of the axes rotation one may proceed with a reference frame, where one axis is parallel to the wave vector. In this reference frame the Reynolds equations contain only 2 coordinates and a stream 
function can be introduced. Derivation of the quasi-linear set of coupled equations for the mean wave fields and harmonics is described in detail in Troitskaya et al. (2012). Its extension to the case of a stratified atmosphere is similar to that described in Part 1 for the case of a single harmonic wave at the water surface.

The solution to the Reynolds equations for 3 velocity components $(u, v, w)$ and density $\rho$ is sought in the form

$$
\begin{aligned}
u= & U_{0}(\eta)+\int\left(\psi_{1 \eta}(\eta) \cos \theta-v_{1}(\eta) \sin \theta\right) \\
& \mathrm{d} A e^{i\left(k\left(\zeta_{1} \cos \theta+\zeta_{2} \sin \theta\right)-\omega t\right)-k \eta-i \phi}, \\
v= & V_{0}(\eta)+\int\left(\psi_{1 \eta}(\eta) \sin \theta+v_{1}(\eta) \cos \theta\right) \\
& \mathrm{d} A e^{i\left(k\left(\zeta_{1} \cos \theta+\zeta_{2} \sin \theta\right)-\omega t\right)-k \eta-i \phi}, \\
w= & -\int i k \psi_{1}(\eta) \mathrm{d} A e^{i\left(k\left(\zeta_{1} \cos \theta+\zeta_{2} \sin \theta\right)-\omega t\right)-k \eta-i \phi}, \\
\rho= & \rho_{0}(\eta)+\int \rho_{1}(\eta) \mathrm{d} A e^{i\left(k\left(\zeta_{1} \cos \theta+\zeta_{2} \sin \theta\right)-\omega t\right)-k \eta-i \phi} .
\end{aligned}
$$

Here, $U_{0}(\eta)$ and $V_{0}(\eta)$ are constituents of a mean velocity field, and $\rho_{0}(\eta)$ is an average density profile.

The wave-induced perturbations of vorticity, stream function and density in the air satisfy the following linearized equations:

$$
\begin{aligned}
& \left(\psi_{0 \eta} \chi_{1}-\psi_{1} \chi_{0 \eta}\right) i k-i k \frac{g}{\rho_{a 0}} \rho_{1} \\
& +i k a \frac{g}{\rho_{a 0}} \frac{\mathrm{d} \rho_{0}}{\mathrm{~d} \eta} e^{-k \eta}-\left(\frac{\mathrm{d}^{2}}{\mathrm{~d} \eta^{2}}-k^{2}\right)\left(\chi_{1} K_{\mathrm{m}}\right) \\
& =-2 K_{m_{\eta}} \psi_{1} k^{2}-2 k^{2} e^{-k \eta}\left(\psi_{0 \eta} K_{m_{\eta}}\right)_{\eta} \\
& \frac{\mathrm{d}^{2} \psi_{1}}{\mathrm{~d} \eta}-k^{2} \psi_{1}=\chi_{1}-2 k e^{-k \eta} \psi_{0 \eta \eta}, \\
& \left(\psi_{0 \eta} \rho_{1}-\psi_{1} \rho_{0 \eta}\right) i k=K_{\rho}\left(\frac{\mathrm{d}^{2}}{\mathrm{~d}^{2}}-k^{2}\right) \rho_{1} \\
& +K_{\rho_{\eta}} \rho_{1 \eta} k^{2}, \\
& \left(\psi_{0 \eta} v_{1}-\psi_{1} \hat{V}_{\eta}\right) i k=K_{\mathrm{m}}\left(\frac{\mathrm{d}^{2}}{\mathrm{~d}^{2}}-k^{2}\right) v_{1}+K_{m_{\eta}} v_{1 \eta} k^{2} .
\end{aligned}
$$

Here, $\psi_{0 \eta}=U_{0}(\eta) \cos \theta+V_{0}(\eta) \sin \theta, \hat{V}=V_{0}(\eta) \cos \theta-$ $U_{0}(\eta) \sin \theta$. In this form, Eqs. (10)-(13) coincide with Eqs. (55)-(57) in Part 1, and all methods developed for investigation of the air turbulent boundary layer are applicable in this case.

The boundary conditions (see Reutov, 1995) are

$$
\begin{array}{ll}
\left.\psi_{1}\right|_{\eta=0}=0, & \left.\psi_{1 \eta}\right|_{\eta=0}=2 \omega, \\
\left.v_{1}\right|_{\eta=0}=0, & \left.\rho_{1}\right|_{\eta=0}=0 .
\end{array}
$$

In the equations for the mean velocity and density components one has to account for the nonlinear terms - the wave momentum fluxes or wave stresses and the mass fluxes. The set of equations for mean vorticity, stream function and density is similar to Eqs. (58)-(60) in Part 1:

$$
\begin{aligned}
& \frac{\mathrm{d}}{\mathrm{d} \eta}\left(K_{\mathrm{m}} \chi_{0}\right)= \\
& k\left[k K_{\mathrm{m} \eta} \operatorname{Re}\left(\psi_{1 \eta}-k \psi_{1}\right) e^{-k \eta}+2 k^{2} e^{-2 k \eta} K_{\mathrm{m} \eta} \psi_{0 \eta}\right]- \\
& -\frac{1}{2} k \frac{\mathrm{d}}{\mathrm{d} \eta} \operatorname{Im}\left(\psi_{1}^{*} \chi_{1}\right)+\frac{1}{2} \frac{g}{\rho_{a 0}} k \frac{\mathrm{d}}{\mathrm{d} \eta} \operatorname{Im}\left(\rho_{1} e^{-k \eta}\right) \\
& =-\frac{\mathrm{d} T}{\mathrm{~d} \eta}, \\
& \frac{\mathrm{d}^{2} \psi_{0}}{\mathrm{~d} \eta^{2}}=\chi_{0}\left(1+k^{2} e^{-2 k \eta}\right)-k e^{-k \eta} \operatorname{Re} \chi_{1}, \\
& \frac{\mathrm{d}}{\mathrm{d} \eta}\left(K_{\rho} \frac{\mathrm{d} \rho_{0}}{\mathrm{~d} \eta}\right)=-\frac{k}{2} \frac{\mathrm{d}}{\mathrm{d} \eta} \operatorname{Im}\left(\rho_{1} \psi_{1}^{*}\right) .
\end{aligned}
$$

The equation for the velocity component, perpendicular to the wind direction, is

$$
\frac{\mathrm{d}}{\mathrm{d} \eta}\left(K_{\mathrm{m}} \frac{d \hat{V}}{\mathrm{~d} \eta}\right)=-\frac{k}{2} \frac{\mathrm{d}}{\mathrm{d} \eta} \operatorname{Im}\left[v_{1} \psi_{1}^{*}\right]=\frac{\mathrm{d}}{\mathrm{d} \eta} \tau_{\perp}(\eta, k, \theta, \omega) .
$$

In quasi-linear approximation the contribution of all surface waves to the mean velocity profile is determined by the momentum fluxes from wind to different harmonics. The wind velocity projections on the $x$ and $y$ axes satisfy the following equations:

$$
\begin{aligned}
& \frac{\mathrm{d}}{\mathrm{d} \eta}\left(K_{\mathrm{m}} \frac{\mathrm{d} U_{0}}{\mathrm{~d} \eta}\right)= \\
& \frac{\mathrm{d}}{\mathrm{d} \eta} \int\left(\tau_{\|}(\eta, k, \theta, \omega) \cos \theta-\tau_{\perp}(\eta, k, \theta, \omega) \sin \theta\right) \\
& k^{2} F(k, \theta, \omega) k \mathrm{~d} k \mathrm{~d} \theta \mathrm{d} \omega, \\
& \frac{\mathrm{d}}{\mathrm{d} \eta}\left(K_{\mathrm{m}} \frac{\mathrm{d} V_{0}}{\mathrm{~d} \eta}\right)= \\
& \quad \frac{\mathrm{d}}{\mathrm{d} \eta} \int\left(\tau_{\|}(\eta, k, \theta, \omega) \sin \theta+\tau_{\perp}(\eta, k, \theta, \omega) \cos \theta\right) \\
& k^{2} F(k, \theta, \omega) k \mathrm{~d} k \mathrm{~d} \theta \mathrm{d} \omega, \\
& \frac{\mathrm{d}}{\mathrm{d} \eta}\left(K_{\rho} \frac{\mathrm{d} \rho_{0}}{\mathrm{~d} \eta}\right)= \\
& \quad-\frac{\mathrm{d}}{\mathrm{d} \eta} \int \frac{k}{2} \operatorname{Im}\left[\rho_{1} \psi_{1}^{*}\right] \\
& F(k, \theta, \omega) k \mathrm{~d} k \mathrm{~d} \theta \mathrm{d} \omega,
\end{aligned}
$$

where $\eta$ corresponds to transformation Eq. (5), $\tau_{\perp}$ is specified by Eq. (18), and from Eqs. (15)-(16) one easily obtains

$\tau_{\|}(\eta, k, \theta, \omega)=-T-k\left(K_{\mathrm{m}} e^{-k \eta} \operatorname{Re}\left(\chi_{1}\right)\right)$.

Equations (19)-(20) correspond to conservation of a vertical flux of the two horizontal momentum projections in a turbulent boundary layer. If tangential turbulent stress far from the surface is directed to $x$, then a conservation law of the 

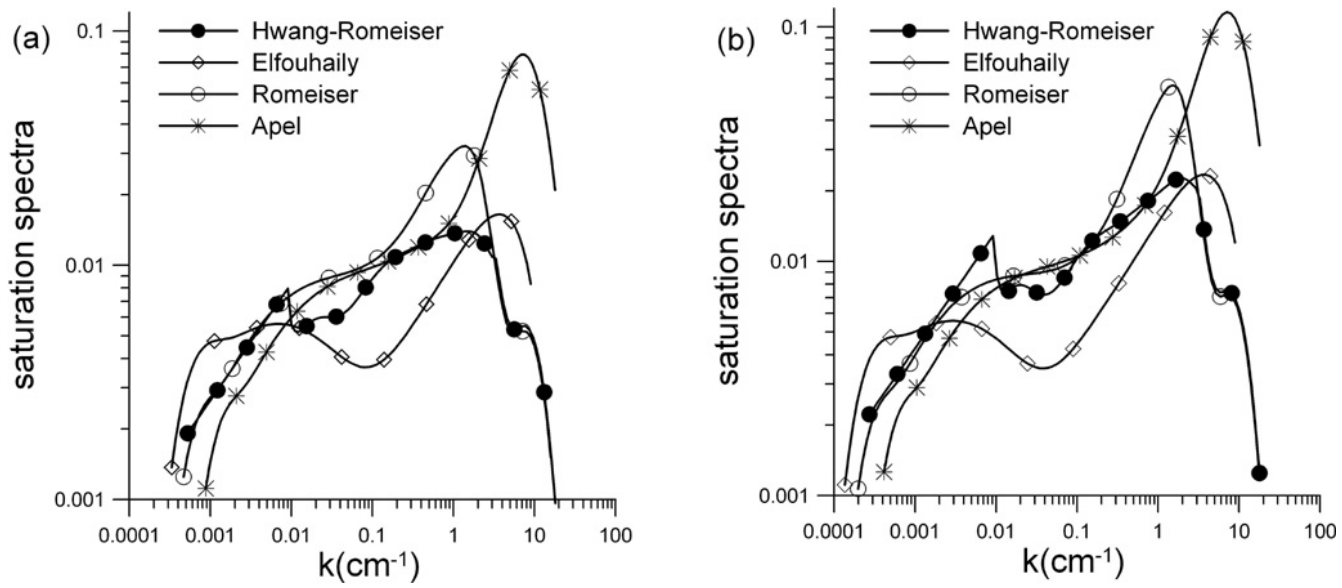

Fig. 1. Model surface wave spectra, friction velocity - (a) $u_{*}=50 \mathrm{~cm} \mathrm{~s}^{-1}$; (b) $u_{*}=80 \mathrm{~cm} \mathrm{~s}^{-1}$, wave age $\Omega=0.84$.

mean momentum components yields

$\tau_{\text {turb }}^{(x)}(\eta)+\tau_{\text {wave }}^{(x)}(\eta)=u_{*}^{2}$,

$\tau_{\text {turb }}^{(y)}(\eta)+\tau_{\text {wave }}^{(y)}(\eta)=0$,

where $u_{*}$ is a friction velocity,

$\tau_{\text {turb }}^{(x)}(\eta)=K_{\mathrm{m}} \frac{\mathrm{d} U_{0}}{\mathrm{~d} \eta}$

$\tau_{\text {wave }}^{(x)}(\eta)=-\int\left(\tau_{\|}(\eta, k, \theta, \omega) \cos \theta-\tau_{\perp}(\eta, k, \theta, \omega) \sin \theta\right)$

$k^{2} F(k, \theta, \omega) k \mathrm{~d} k \mathrm{~d} \theta \mathrm{d} \omega$,

$\tau_{\text {turb }}^{(y)}(\eta)=K_{\mathrm{m}} \frac{\mathrm{d} V_{0}}{\mathrm{~d} \eta}$

$\tau_{\text {wave }}^{(y)}(\eta)=-\int\left(\tau_{\|}(\eta, k, \theta, \omega) \sin \theta+\tau_{\perp}(\eta, k, \theta, \omega) \cos \theta\right)$

$k^{2} F(k, \theta, \omega) k \mathrm{~d} k \mathrm{~d} \theta \mathrm{d} \omega$.

If the wave spectrum is symmetrical with respect to the wind direction, then Eq. (24) is automatically fulfilled.

Integrating of (21) with respect to $\eta$ gives conservation of the mass flux:

$\tau_{\rho \text { turb }}(\eta)+\tau_{\rho \text { wave }}(\eta)=\rho_{*} u_{*}$

where $\rho_{*}$ is the turbulent scale of density, characterizing the mass flux,

$\tau_{\rho \mathrm{turb}}(\eta)=K_{\rho} \frac{\mathrm{d} \rho_{0}}{\mathrm{~d} \eta} ;$

$\tau_{\rho \text { wave }}(\eta)=\int \frac{k}{2} \operatorname{Im}\left[\rho_{1} \psi_{1}^{*}\right] F(k, \theta, \omega) k \mathrm{~d} k \mathrm{~d} \theta \mathrm{d} \omega$

Since the wave-induced momentum and the mass fluxes decrease with the distance from the water surface, the boundary conditions for the mean velocity and density then follow from $(23,24,29)$ :

$$
\begin{aligned}
& \left.K_{\mathrm{m}} \frac{\mathrm{d} U_{0}}{\mathrm{~d} \eta}\right|_{\eta \rightarrow \infty}=u_{*}^{2},\left.\quad K_{\mathrm{m}} \frac{\mathrm{d} V_{0}}{\mathrm{~d} \eta}\right|_{\eta \rightarrow \infty}=0, \\
& \left.K_{\rho} \frac{\mathrm{d} \rho_{0}}{\mathrm{~d} \eta}\right|_{\eta \rightarrow \infty}=\rho_{*} u_{*} .
\end{aligned}
$$

The coupled systems for the wave disturbances Eqs. (10)(13) with the boundary conditions in Eq. (14) and for the mean fields Eqs. (19)-(21) with the boundary conditions in Eq. (31) were solved numerically. Vertical profiles of velocity, density and fluxes of momentum and mass were obtained from these calculations and exchange coefficients for neutral atmosphere $C_{\mathrm{D}_{10 \mathrm{~N}}}$ and $C_{\rho_{10 \mathrm{~N}}}$ (see Zeng et al., 1998, and Part 1) were then calculated.

\section{Surface elevation spectra}

According to Eqs. (19)-(21), nonlinear additives to the mean velocity and density profiles are determined by the wave number-frequency spectrum of surface waves; hence, a spectrum is an important part of the model. First of all, supposing that frequencies and wave numbers of surface waves obey a linear dispersion relation, a wave number-frequency spectrum can then be written in the form

$F(\boldsymbol{k}, \omega)=S(\boldsymbol{k}) \delta(\omega-\omega(\boldsymbol{k}))$.

This assumption is justified for long waves, but in the short-wave part of a spectrum the contribution of the bound waves, which are the harmonics of the wind waves, may be considerable. Their dispersion relation may differ significantly from the usual linear one. However, phase velocities of the short waves are usually smaller than wind speed; in this case the dispersion relation for the surface perturbations has weak influence on air-sea momentum exchange.

We investigated the sensitivity of the drag coefficient and the mass transfer coefficient to the wave spectrum. In the 


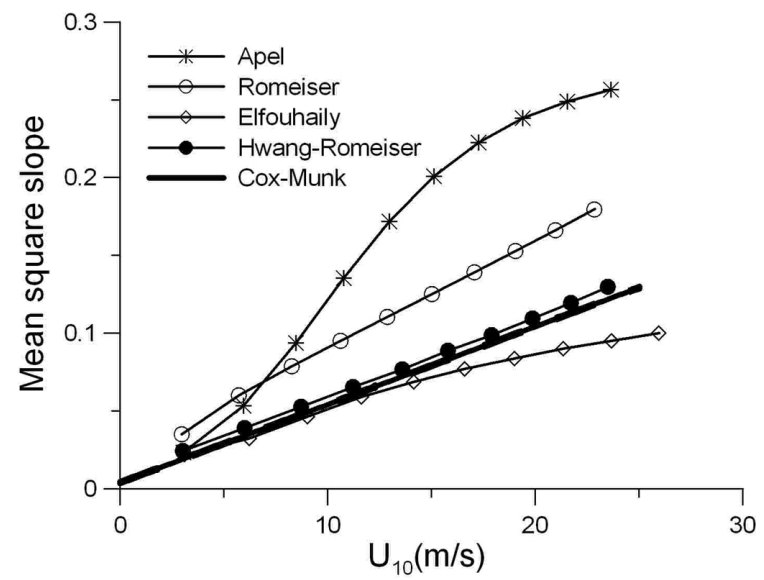

Fig. 2. Mean square slope dependence on wind speed.

calculations several model spectra were used: Apel (1994), Romeiser et al. (1997), Hwang et al. (1996, 2001a, b), Hwang (1997, 2005) and Hwang and Wang (2004). They are shown in Fig. 1 for a developed sea at wind speeds 50 and $80 \mathrm{~cm} \mathrm{~s}^{-1}$. The expressions for these spectra are given in Appendix A.

In the calculations the upper bound of the spectrum was $20 \mathrm{~cm}^{-1}$ (corresponding to the wave length of $3.14 \mathrm{~mm}$ ); moreover, all the saturated model spectra decay exponentially. Hence, the mean square slope is fully determined by this spectral interval. Figure 2 shows the mean square slopes for the different spectra as the functions of wind speed. The same figure shows the mean square slope, which in this case is obtained by Cox and Munk (1954) from measurements of a sun glitter. It can be concluded that Hwang's spectrum (see Eq. A4), completed by Romeiser spectrum (Eq. A3) for $k>3.16 \mathrm{~cm}^{-1}$, provides the best fit curve to Cox's and Munk's. Note that these spectra are obtained from the approximations of the field measurements data, while other model spectra are obtained from the laboratory experiments.

\section{An appropriate model of the turbulent transfer coefficients}

To solve the set of Eqs. (15)-(18) and (19)-(21) numerically, it is necessary to choose the right model of turbulent transfer coefficients. The expressions were chosen similarly to Part 1 :

$K_{\mathrm{m}}(\eta)=\frac{\nu_{\mathrm{a}} f\left(\eta / z_{\nu}\right)}{\Phi_{\mathrm{m}}(\eta / L)}, \quad K_{\rho}(\eta)=\frac{\nu_{\mathrm{a}} f_{\rho}\left(\eta / z_{\nu}\right)}{\Phi_{\rho}(\eta / L)}$.

Here, $v_{\mathrm{a}}$ is air molecular viscosity, $z_{v}$ is a viscous sublayer width, $L$ is the Obukhov length scale, and $\Phi_{\mathrm{m}}(z / L)$, $\Phi_{\rho}(z / L)$ are the universal functions of dimensionless height (we used the empirical approximations from Zeng et al., 1998; see also Part 1$)$. We adopt $f\left(\eta / z_{v}\right)$ obtained by Smol'yakov (1973) for a turbulent flow over smooth plate: $f\left(\eta / z_{v}\right)=1+\frac{\kappa \eta}{z_{v}}\left(1-\exp \left\{-\left(\frac{\eta}{z_{v} l_{\mathrm{m}}}\right)^{2}\right\}\right)$,

where $l_{\mathrm{m}}=22.4$. The similar expression holds for $f_{\rho}\left(\eta / z_{v}\right)$ :

$f_{\rho}\left(\eta / z_{v}\right)=v_{\mathrm{a}}\left(\frac{1}{P r}+\frac{\kappa \eta}{P r_{\mathrm{t}} z_{v}}\left(1-\exp \left\{-\left(\frac{\eta}{z_{v} l_{\rho}}\right)^{2}\right\}\right)\right)$.

Here, $\operatorname{Pr}=v_{\mathrm{a}} / v_{\rho}$ is the Prandtl number; $P r_{\mathrm{t}}=K_{\mathrm{m}} / K_{\rho}$ is the turbulent Prandtl number in neutral and near-neutral stratification, $l_{\rho}=31.7$. For a detailed discussion see Part 1 .

In the presence of surface waves, there is an additional dimensionless function, normalized wave momentum flux $\tau_{\text {wave }}(\eta) / u_{*}^{2}$, where $\tau_{\text {wave }}(\eta)=$ $\sqrt{\left(\tau_{\text {wave }}^{(x)}(\eta)\right)^{2}+\left(\tau_{\text {wave }}^{(y)}(\eta)\right)^{2}}$ is the magnitude and $\tau_{\text {wave }}^{(x),(y)}(\eta)$ are $x$ and $y$ components of the wave momentum flux given by Eqs. (26) and (28). In this case turbulent transfer coefficients may depend on this function, i.e.,

$\begin{aligned} K_{\text {mwave }}(\eta) & =\frac{\nu_{\mathrm{a}} f\left(\eta / z_{\nu}, \tau_{\text {wave }}(\eta) / u_{*}^{2}\right)}{\Phi_{\mathrm{m}}(\eta / L)}, \\ K_{\rho \text { wave }}(\eta) & =\frac{\nu_{\mathrm{a}} f_{\rho}\left(\eta / z_{\nu}, \tau_{\text {wave }}(\eta) / u_{*}^{2}\right)}{\Phi_{\rho}(\eta / L)} .\end{aligned}$

From the calculations it follows that the wave-induced mass flux is small; hence, it may be omitted in a parameterization of turbulent transfer. In Troitskaya and Rybushkina (2008) the eddy viscosity dependence on waves was accounted for as follows. Instead of $z_{\nu}=$ $v_{\mathrm{a}} / u_{*}$ the authors used $z_{*}=v_{\mathrm{a}} / \sqrt{\tau_{\text {turb }}(\eta)}$, where $\tau_{\text {turb }}(\eta)=$ $\sqrt{\left(\tau_{\text {turb }}^{(x)}(\eta)\right)^{2}+\left(\tau_{\text {turb }}^{(y)}(\eta)\right)^{2}}$ is a magnitude of a turbulent momentum flux; from Eqs. (23) and (24), $\tau_{\text {turb }}(\eta)=u_{*}^{2}-$ $\tau_{\text {wave }}(\eta)$. In this case turbulent transfer coefficients are given by Eqs. (31)-(35), with a substitution $\eta / z_{v} \rightarrow \eta / z_{*}$, where $\eta / z_{*}=\eta_{*} \sqrt{1-\tau_{\text {wave }}(\eta) / u_{*}^{2}}$. A similar approximation was used in Makin and Mastenbroek (1996) and Makin and Kudryavtsev (1999).

Thus, we have to choose an appropriate closure model (CM) that gives an adequate description of the exchange coefficients. Both of the models considered here are given by the expressions Eqs. (33)-(35) for the eddy viscosity and the eddy conductivity. The difference is that in the first model (CM1), the viscous scale is determined by $u_{*}$ corresponding to the whole momentum flux, while in the second (CM2), $u_{*} \sqrt{1-\tau_{\text {wave }}(\eta) / u_{*}^{2}}$ is corresponding to the turbulent momentum flux. Since these models are equivalent from the point of view of scaling theory, only comparison with experimental data allows for choosing between them. Further in Sect. 4.1 we discuss results of the calculations of $C_{\mathrm{D}}$ and $C_{\rho}$ 


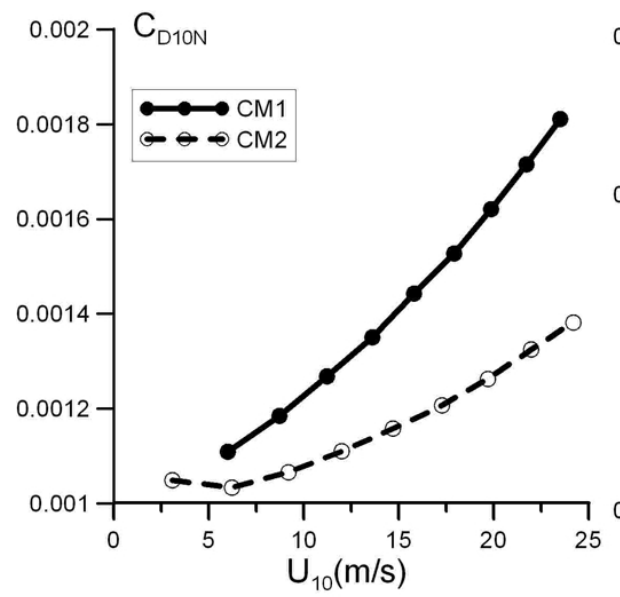

(a)

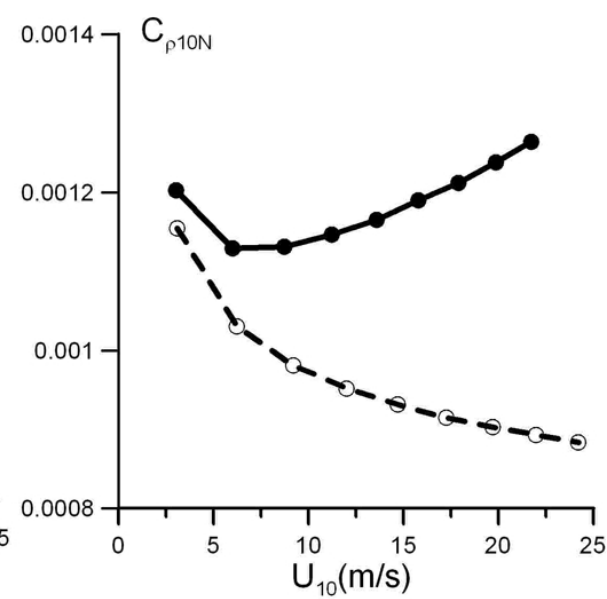

(b)

Fig. 3. Dependencies of a drag coefficient (a) and a mass exchange coefficient (b) on wind speed.

for both CMs, which show that better agreement is achieved with CM1. This conclusion is also confirmed on the basis of comparison with the laboratory experiment in the wind-wave flume. The details are described in the following subsections.

\subsection{Comparison to TOGA/COARE experimental data. Temperature and wind velocity profiles above a wavy water surface}

Wind speed dependencies of $C_{\mathrm{D}_{10 \mathrm{~N}}}$ and $C_{\rho 10 N}$, obtained within the framework of the two closure models, are shown in Fig. 3. For calculations we used the modified Hwang spectrum (Hwang, 2005 - see Eq. A14), completed by Romeiser spectrum (Romeiser et al., 1997), which corresponds best to the experimental data of Cox and Munk (1954).

It can be seen from Fig. 3a that the dependence $C_{\mathrm{D}_{10 \mathrm{~N}}}\left(U_{10}\right)$ is weaker for CM2, and Fig. 3b shows that CM2 gives a decreasing dependence $C_{\rho_{10 \mathrm{~N}}}\left(U_{10}\right)$. The last result contradicts the field observations, approximated by COARE 3.0 algorithm and the data from Brut et al. (2005).

In order to illustrate the difference between the two closure models, let us consider the mean velocity and density profiles, turbulent fluxes and turbulent transfer coefficients calculated within the models. Figure $4 \mathrm{a}-\mathrm{f}$ show mean velocity (a) and density (b) profiles, turbulent momentum (c) and mass (d) fluxes, turbulent momentum (e) and mass (f) transfer coefficients in weak stable stratification and weak wind, when $\rho_{*} / \rho_{0}=0.5 / 300$ and $u_{*}=10 \mathrm{~cm} \mathrm{~s}^{-1}$. It can be concluded from Fig. $4 \mathrm{~d}$ and e that, in the presence of waves, CM2 gives smaller turbulent transfer coefficients $K_{\rho}$ and $K_{\mathrm{m}}$ than CM1. This is the consequence of the turbulent momentum flux decrease close to the surface (Fig. 4c), as this flux characterizes the eddy viscosity within the framework of CM2. Decrease of the turbulent tangential stress close to the surface due to wind-to-waves momentum transfer results in decrease of wind speed and increase of the drag as compared to the flow above the smooth surface (see Fig. 4a). Within the framework of CM2 this effect is weaker (compare solid and dotted curves in Fig. 4a) due to the decrease of the eddy viscosity.

It is interesting to notice the effect of the decreasing turbulent mass flux close to the surface (see Fig. 4d) due to the wave mass flux. This effect has already been discussed in Part 1. It is known that the resonant interaction of waves with stratified shear flows in the viscous and thermal-transfer media leads to the irreversible reduction of the density gradient in the region with closed streamlines - the critical layers (see, e.g., Maslowe, 1972; Haberman, 1973; Troitskaya, $1991)$ - which leads to a positive wave mass-flux $\tau_{\rho \text { wave }}(\eta)$ in Eq. (29) and to a reduction of the average density gradient. In turn, it leads to a reduction of the density difference between the sea surface and the reference level. This effect increases with a decreasing coefficient of heat conductivity (see Troitskaya, 1991), meaning it is most pronounced in a condition of light winds and strong stratification of the surface layer of the atmosphere where the coefficients of turbulent mass transfer are reduced. The consequence of the decrease of a turbulent mass flux is the decrease of the mean density difference.

In this study the total decrease of the turbulent mass flux does not exceed $15 \%$; this is a consequence of big enough $K_{\rho}$. For CM2 this effect is more significant due to less $K_{\rho}$. For CM1, the consequence of the decreasing turbulent mass flux is the decreasing density difference. For CM2 this effect is compensated: the density difference increases because of the decreasing turbulent mass transfer coefficient (compare solid and dotted curves in Fig. 4b).

Analogous dependencies for the wind friction velocity $u_{*}=50 \mathrm{~cm} \mathrm{~s}^{-1}$ are shown in Fig. $5 \mathrm{a}-\mathrm{f}$. Note that the common peculiarities of the closure models stay for stronger winds. One of the differences is the significant reduction of 


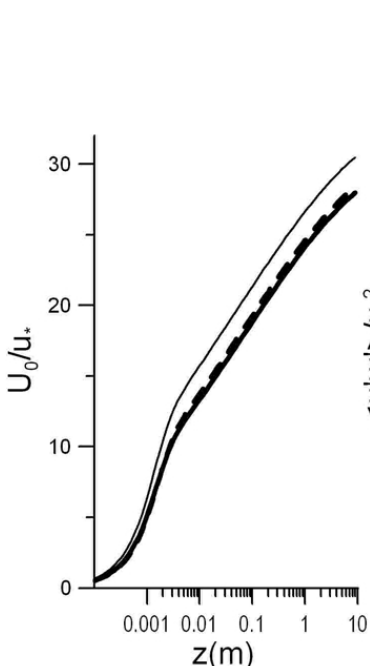

(a)

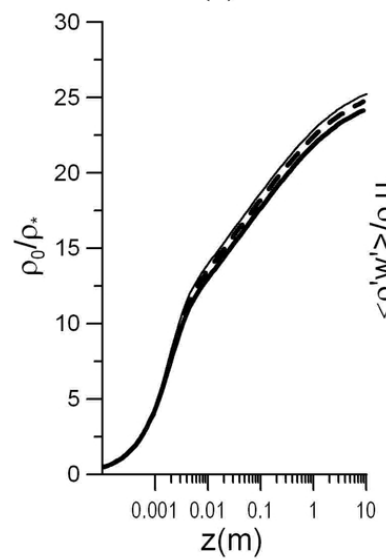

(b)

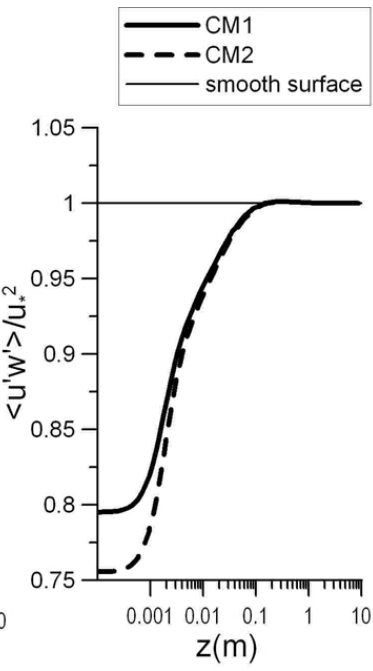

(c)

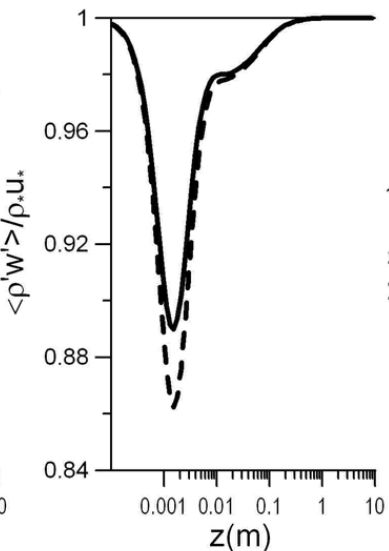

(d)

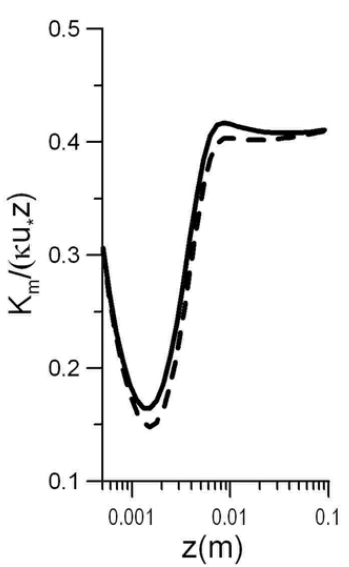

(e)

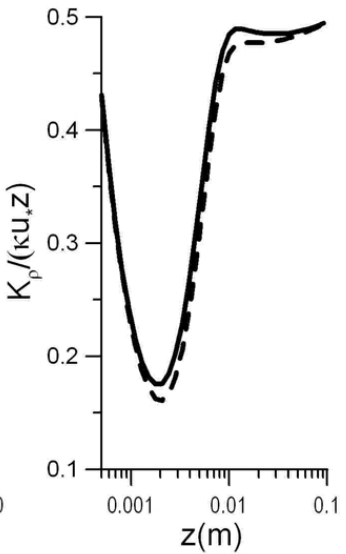

(f)

Fig. 4. Mean velocity (a) and density (b) profiles, turbulent momentum (c) and mass (d) fluxes, normalized turbulent momentum (e) and mass (f) transfer coefficients. Friction velocity $-10 \mathrm{~cm} \mathrm{~s}^{-1}$.

a turbulent momentum flux close to the surface in strong wind (Fig. 5c), causing decrease of the eddy viscosity for CM2 (Fig. 5e). Reduction of a turbulent mass flux is less pronounced than for weak wind due to the increase of a turbulent transfer coefficient with wind speed (Fig. 5d). As a result, within the framework of CM1, density difference in the case of waves is almost indistinguishable from the case of the flow above the smooth surface (Fig. 5b). At the same time, CM2 gives significant increase in density leap in the presence of waves as compared to the flow above the smooth surface (Fig. 5b).

The differences in the velocity and density profiles for the two models explain the differences in the turbulent exchange coefficients. As follows from Fig. 3a, $C_{\mathrm{D}_{10 \mathrm{~N}}}$ grows faster with $U_{10 N}$ for CM1. This is also confirmed by the velocity profiles calculated for both models (Figs. 4a and 5a); CM1 gives smaller $U_{10 \mathrm{~N}}$ in the same conditions due to the larger eddy viscosity. The air density difference $\Delta \rho_{10 \mathrm{~N}}$ between the surface and the standard height $10 \mathrm{~m}$ within the framework of
CM1 is somewhat less than for a smooth surface due to the influence of the wind stresses on turbulent mass transfer. This effect, however, cannot be verified experimentally because the reduction does not exceed $1 \%$. Within the framework of CM2, density difference $\Delta \rho_{10 \mathrm{~N}}$ is larger due to the reduction of the turbulent mass transfer coefficient.

As a result, CM1 gives the growing exchange coefficient $C_{\rho_{10 \mathrm{~N}}}$ due to both increasing drag coefficient and ratio $\rho_{*} / \Delta \rho_{10 \mathrm{~N}}$ (see Eq. 40 in Part 1). Within the framework of $\mathrm{CM} 2$, the ratio $\rho_{*} / \Delta \rho_{10 \mathrm{~N}}$ decreases with wind speed because of the effective reduction of the turbulent transfer coefficient for strong winds (see Figs. $4 \mathrm{f}$ and $5 \mathrm{f}$ ). At the same time, growth of $C_{\mathrm{D}_{10 \mathrm{~N}}}$ with wind speed $U_{10 \mathrm{~N}}$ is not enough for the compensation of the decreasing $\rho_{*} / \Delta \rho_{10 \mathrm{~N}}$. Consequently, $C_{\rho_{10 \mathrm{~N}}}$ decreases with wind speed.

Thus, calculations within the framework of CM1 give better qualitative agreement with experimental data (Fairall et al., 2003; Ocampo-Torres et al., 1994; Brut et al., 2005), 


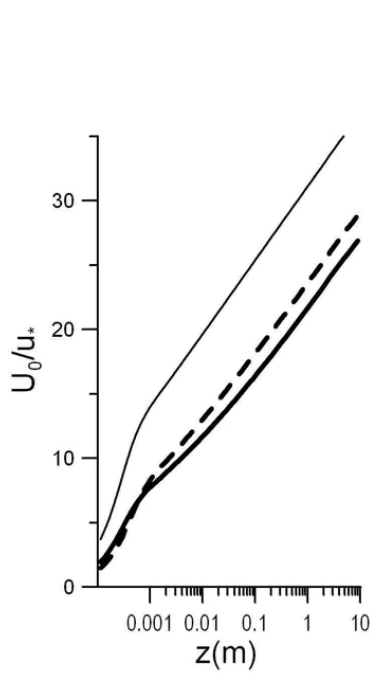

(a)

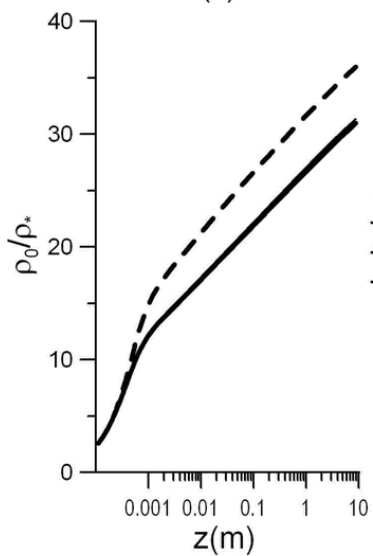

(b)

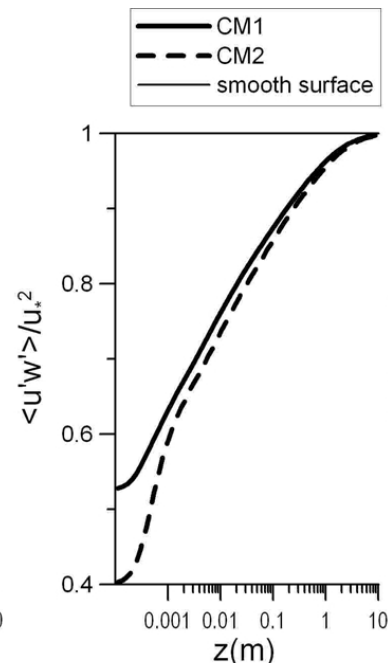

(c)

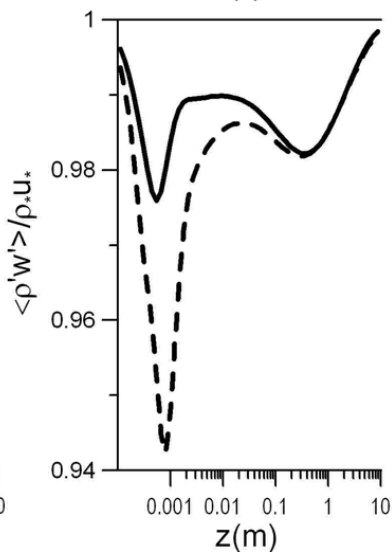

(d)

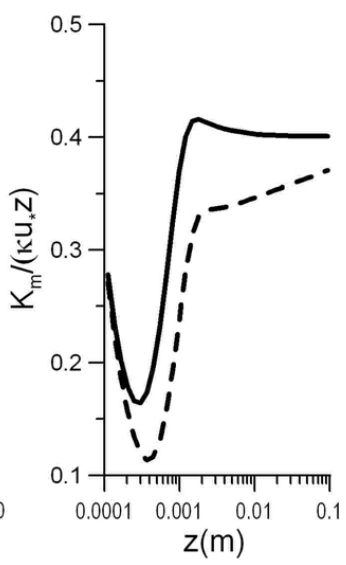

(e)

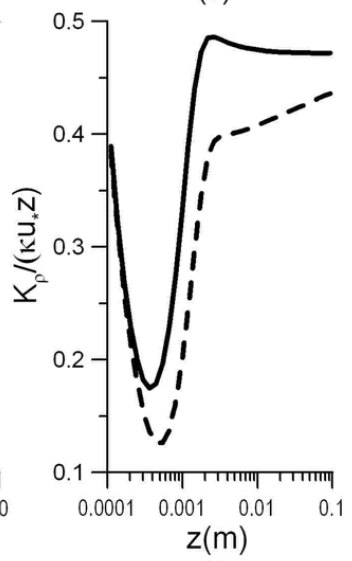

(f)

Fig. 5. Mean velocity (a) and density (b) profiles, turbulent momentum (c) and mass (d) fluxes, normalized turbulent momentum (e) and mass (f) transfer coefficients. Friction velocity $-50 \mathrm{~cm} \mathrm{~s}^{-1}$. The thin and thick solid curves are indistinguishable in (b).

demonstrating the growing dependencies of $C_{\rho_{10 \mathrm{~N}}}$ on wind speed.

\subsection{Comparison to the experimental data from the wind-wave flume of IAP RAS}

To select the model of the turbulent exchange coefficients, we also used data from the laboratory experiments directed to study stratified turbulent boundary layer above the water surface. The experiments investigated the effects of surface waves on turbulent momentum and mass transfer in a stratified boundary layer. The experimental setup (Fig. 6) was designed at the wind-wave Large Thermally Stratified Tank (LTST) of IAP RAS (for detailed description of this experiment flume facility see Troitskaya et al., 2012).

Temperature and wind speed at the channel entrance were controlled using a hot-film anemometer. The accuracy of the measurements was within $0.02{ }^{\circ} \mathrm{C}$ for temperature and within
$10 \mathrm{~cm} \mathrm{~s}^{-1}$ for velocity in the whole range of wind speeds in the experiment.

To measure characteristics of the airflow in the work section (at $7 \mathrm{~m}$ distance from the entrance), we used a pneumometric Pitot tube (connected to the differential pressure gauge MKS Baratron $226 \mathrm{AD}$ ) along with a hot-film anemometer similar to the one described above. They were jointed and positioned at the scanning system. Both sensors had the same diameter and the sensing head of the anemometer was at the same height as the inlet of the Pitot tube. This scheme provided measurements of temperature and velocity on the same level. The hot-film anemometer was placed behind the Pitot tube and was used for temperature measurements only. We carried out a test series of experiments, which showed no interference of the sensors on their experimental data. The difference between data from measurements for the cases of separate and combined use of the sensors did not exceed their error range. The accuracy of velocity measurement by Pitot tube is $5 \mathrm{~cm} \mathrm{~s}^{-1}$ in the whole range of wind speeds. 


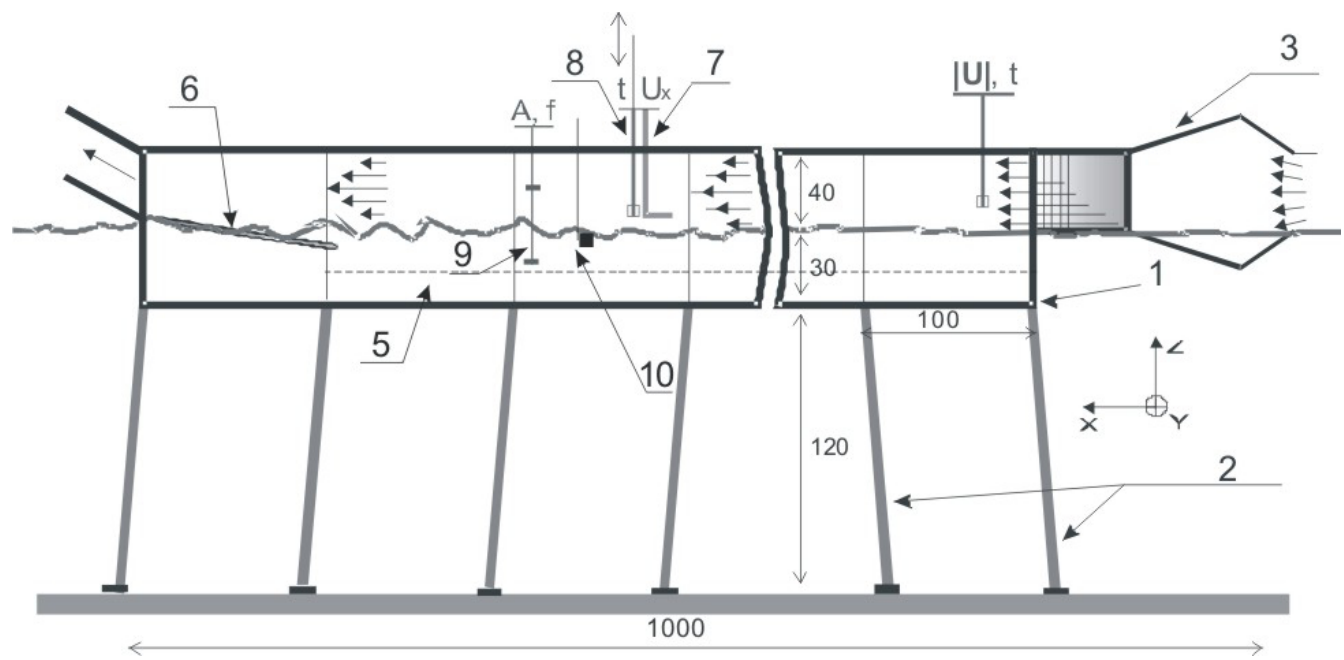

Fig. 6. Experimental setup in the wind-wave LTST of IAP RAS. The sizes are in $\mathrm{cm}$. (1) Wind-wave channel body, (2) wind-wave bearings, (3) convergent - diffusion section with a honeycomb, (4) hot-film anemometer at the entrance, (5) a net along the channel installed on the different depths, (6) wave absorber, (7) Pitot tube on a scanning system, (8) anemometer, (9) three-channel wire wave-gauge, (10) a sensor for water temperature measurements.

The scanning began from different heights of water level, depending on the parameters of surface waves. The minimum height was $10 \mathrm{~mm}$ from the undisturbed water surface. The data accusation time for each horizon was $1 \mathrm{~min}$. The velocity and temperature profiles were measured twice during the experiment.

To regulate wave amplitude independently of the wind, a polyethylene net with a filament width of $0.25 \mathrm{~mm}$ and a mesh size $1.6 \times 1.6 \mathrm{~mm}$ was put under water at $3 \mathrm{~mm}$ depth from the undisturbed water level along the whole channel. To provide the uniform tension and the same depth along the channel we used the underwater wire ( $3 \mathrm{~mm}$ diameter) brackets put at a distance of $1 \mathrm{~m}$ from each other. The deviation of the net location by depth was within $1.5 \mathrm{~mm}$. The net provided the effective attenuation of surface waves dependent on its depth in the whole range of wind speeds, including strong winds (up to $17 \mathrm{~m} \mathrm{~s}^{-1}$ or equivalent $30 \mathrm{~m} \mathrm{~s}^{-1}$ for the reference level $10 \mathrm{~m}$ ). We did not perform experiments for higher wind speeds because of the spray dropping on the anemometers.

A water temperature sensor was put near a wave gauge. It was placed at the same depth of $2.5 \mathrm{~cm}$ from the undisturbed water level for all experiments with the net. When the net was not used, the depth of a sensor location increased with increasing wind speed to provide $3 \mathrm{~cm}$ depth from the mean level of waves' troughs in the working section. The accuracy of this sensor was $0.05^{\circ} \mathrm{C}$.

Stable air stratification near the water surface was provided by the temperature difference between water and air. Average water temperature during the whole experimental series was $14.8^{\circ} \mathrm{C}$. The deviation from this value was within $0.2{ }^{\circ} \mathrm{C}$ due to the large reservoir of the LTST. Mean air tem- perature at the entrance of the channel was $22.9^{\circ} \mathrm{C}$, but varied significantly (the deviation was $0.8^{\circ} \mathrm{C}$ ) for two reasons. First, air temperature at the LTST entrance depends on the speed of fan rotation. Second, the temperature in the lab changes from day to day in spite of the special climate systems. Thus, the experimental data were scaled by the entrance temperatures.

The experiments were performed for 2 different wind speeds with and without a net. The wind speeds at the axis of the LTST in the working section were $13.9 \mathrm{~m} \mathrm{~s}^{-1}$ and $16.5 \mathrm{~m} \mathrm{~s}^{-1}$.

It is clearly seen from Fig. 7 that wind velocity profiles are significantly different for the cases with and without a net. In contrast to the velocity profiles, the temperature profiles are almost independent of the wave amplitudes determined by the net position. This corresponds to CM1: the form of the wave surface does not influence the mass fluxes. This result allows choosing the model CM1 with the wave-independent turbulent transfer coefficients for further analysis.

\subsection{Sensitivity of the model to the wave spectrum}

One of the most important constituents of the model is the surface wave spectrum when determining the wave momentum and the mass fluxes (see Eqs. 19-21). According to Hwang (2005), waves with the wave lengths from centimeters to $1 \mathrm{~m}$ are the ones that contribute the most to surface roughness. Indeed, let us consider the contributions to surface roughness of wave perturbations induced by the separate wave harmonics for the different model spectra. The wind speed profile over a wavy water surface can be represented as a superposition of the logarithmic velocity profile over a smooth surface and the constant negative additive (see, e.g., 

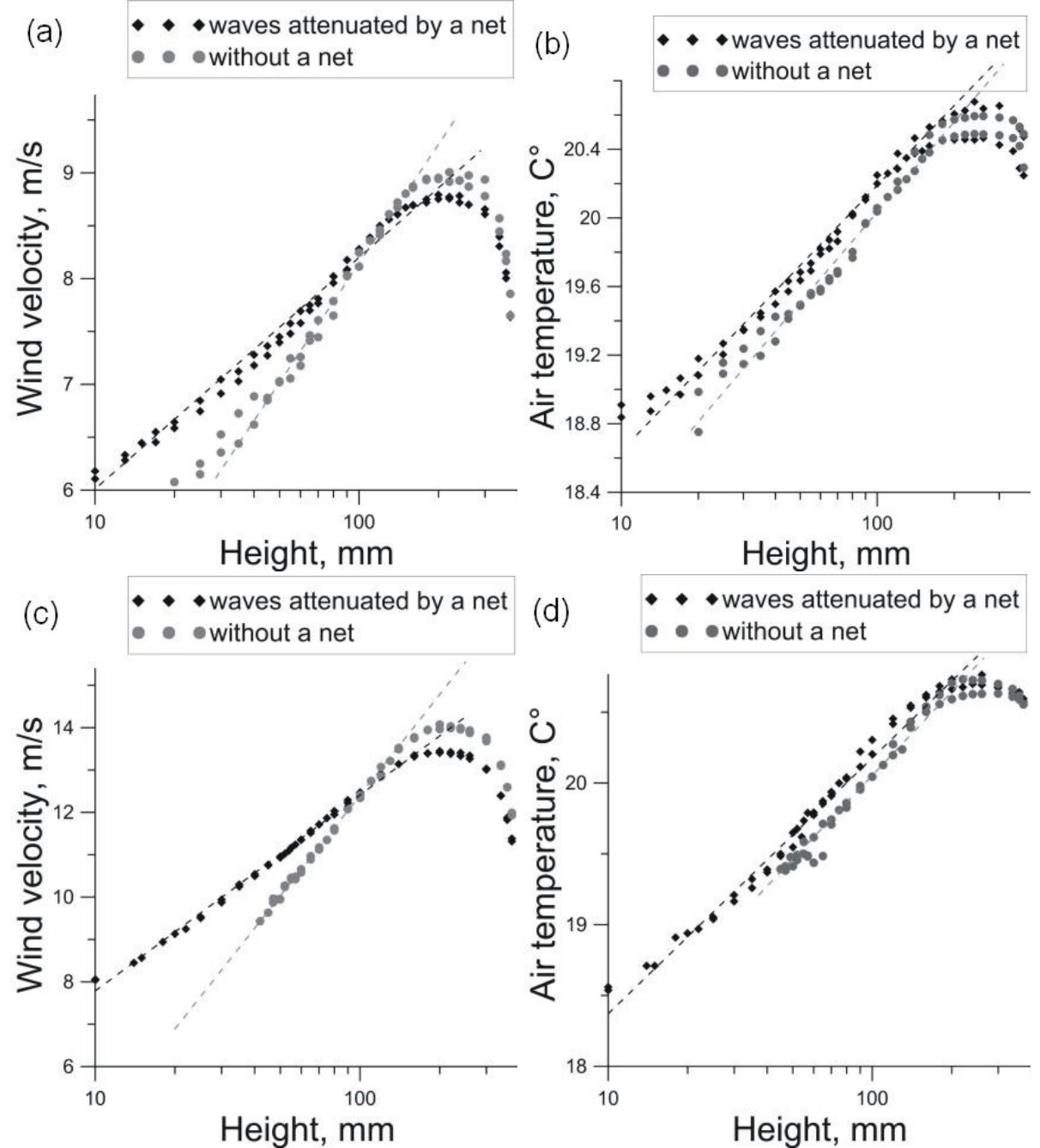

Fig. 7. Wind velocity (a) and (c) and temperature (b) and (d) vertical profiles measured for wind speeds on the axis of the LTST in the working section: $13.9 \mathrm{~m} \mathrm{~s}^{-1}(\mathbf{a}, \mathbf{b}), 16.5 \mathrm{~m} \mathrm{~s}^{-1}$ (c, d).

Eq. 25 from Part 1). The contribution of the separate wave harmonics to this additive is shown in Fig. 8a and b:

$\Delta u(k)=\int_{k_{\mathrm{p}}}^{k} \int_{-\pi}^{\pi} k \mathrm{~d} k \mathrm{~d} \theta \int_{0}^{\infty} \frac{\hat{\tau}_{\mathrm{wave}}(z, k, \theta)}{\mathrm{K}_{\mathrm{m}}(z)} \mathrm{d} z$,

where $\hat{\tau}_{\text {wave }}(z, k, \theta)$ is a wave-induced momentum flux in the air, corresponding to the harmonic wave with wave number $k$, propagating at the angle $\theta$ to the wind direction. The expression for $\hat{\tau}_{\text {wave }}(z, k, \theta)$ is given by the integrand in Eq. (19) while the formulas for surface spectra are specified in Appendix A.

Figure $8 \mathrm{a}$ and $\mathrm{b}$ differ by the value of $u_{*}$, which is equal to $50 \mathrm{~cm} \mathrm{~s}^{-1}$ in Fig. 8 a and to $80 \mathrm{~cm} \mathrm{~s}^{-1}$ in Fig. 8b. The difference in "roughness structure" for the different spectra can be seen from these figures. In particular, Apel spectrum (Apel, 1994) causes underestimation of the harmonics with long wave lengths, and overestimation of the high frequency constituents. For the Hwang's spectrum (Hwang, 2005) (see Appendix A), completed by the Romeiser spectrum (Romeiser et al., 1997) (Eq. A3), $97 \%$ of the sea roughness corresponds to the harmonics with wave numbers less than $4 \mathrm{~cm}^{-1}$.

The coefficients of momentum and mass exchange were calculated within the quasi-linear model according to their definitions.

$C_{\mathrm{D}_{10 \mathrm{~N}}}=\frac{u_{*}^{2}}{U_{10 \mathrm{~N}}^{2}} ; \quad C_{\rho_{10 \mathrm{~N}}}=\frac{\rho_{*} u_{*}}{\Delta \rho_{10 \mathrm{~N}} U_{10 \mathrm{~N}}}$.

Dependencies $C_{\mathrm{D}_{10 \mathrm{~N}}}\left(U_{10 \mathrm{~N}}\right)$ and $C_{\rho_{10 \mathrm{~N}}}\left(U_{10 \mathrm{~N}}\right)$, calculated within the framework of the quasi-linear model, are shown in Fig. 9a and b, respectively. Calculations were performed for a developed sea $\left(\Omega=U_{10} / c=0.84\right)$ with near-neutral stratification, and the turbulent Prandtl number was 0.85 , in accordance with Monin and Yaglom (1992). These parameters were chosen for comparison with the available experimental 


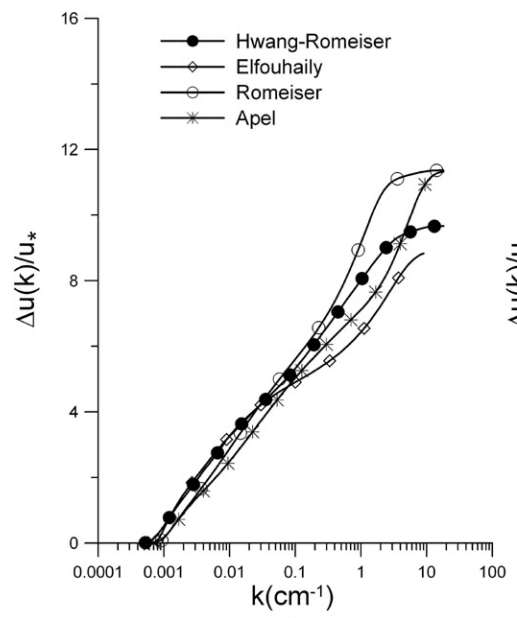

(a)

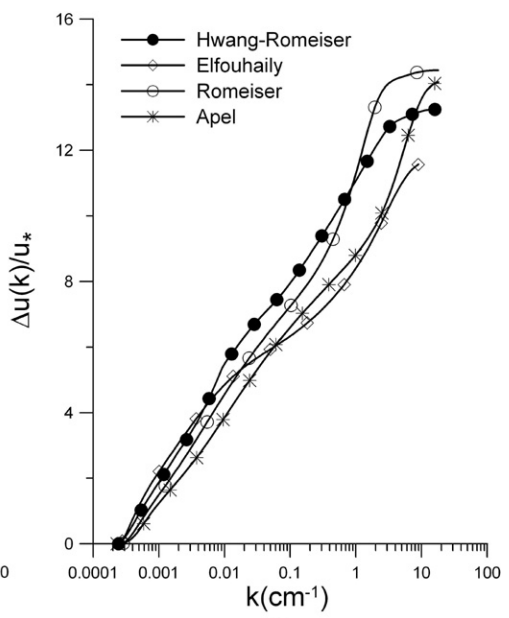

(b)

Fig. 8. Harmonics' contributions to the wave momentum flux on the surface for $u_{*}=50 \mathrm{~cm} \mathrm{~s}^{-1}$ (a) and $u_{*}=80 \mathrm{~cm} \mathrm{~s}^{-1}$ (b).

data. We used TOGA/COARE 3.0 approximations of dependencies $C_{\mathrm{D}_{10 \mathrm{~N}}}\left(U_{10 \mathrm{~N}}\right)$ and $C_{\rho_{10 \mathrm{~N}}}\left(U_{10 \mathrm{~N}}\right)$ published in Fairall et al. (2003).

For quantitative comparison of calculations with experimental data we found relative errors of the model $\varepsilon_{\mathrm{D}}$ and $\varepsilon_{\rho}$ using the following formulas:

$\varepsilon_{D, \rho}=\left(\frac{1}{N} \sum_{n=1}^{N} \frac{\left(C_{\mathrm{D}, \rho 10 N}^{\text {calc }}-C_{\mathrm{D}, \rho 10 N}^{\text {coare }}\right)^{2}}{\left(C_{\mathrm{D}, \rho 10 N}^{\text {coare }}\right)^{2}}\right)^{1 / 2}$,

where index (calc) indicates calculations data, and index (coare) correspond to TOGA/COARE 3.0 data (Fairall et al., 2003). The estimates of the relative errors are shown in Table 1 for wind speeds $U_{10 \mathrm{~N}}$ less than $14 \mathrm{~m} \mathrm{~s}^{-1}$ and $18 \mathrm{~m} \mathrm{~s}^{-1}$ for comparison. It can be seen from Table 1 that better agreement with TOGA/COARE 3.0 data is achieved for Hwang-Romeiser, Romeiser and Apel spectra (error about $10 \%$ for $C_{\mathrm{D}_{10 \mathrm{~N}}}$ and less than $10 \%$ for $C_{\rho_{10 \mathrm{~N}}}$ ). The Elfouhaily spectrum gives values of $C_{\mathrm{D}_{10 \mathrm{~N}}}$ underestimated by about $30 \%$. However, only Hwang-Romeiser's and Elfouhaily spectra are in agreement with Cox and Munk (1954) data, but both Romeiser's and Apel's spectra significantly overestimate mean square slope in comparison with Cox and Munk (1954).

At the same time it can be seen from Fig. 9a that for all spectra the model is in good agreement with the experimental data for wind speed $U_{10 \mathrm{~N}}<10 \mathrm{~m} \mathrm{~s}^{-1}$. For higher winds the model underestimates dependencies $C_{\mathrm{D}_{10 \mathrm{~N}}}$ on wind speed in comparison with the data by Fairall et al. (2003). Given the high sensitivity of the surface drag coefficient to the "structure" of the roughness described by the spectrum of the surface waves, it can be assumed that these differences are due to inaccuracies in the determination of the wind-wave spectra. Indeed, as shown in Fig. 8, the short waves make the main contribution to surface roughness. At the same time, measurement of short waves is a very difficult problem, especially at high wind speeds. Thus, the experimental data for the Hwang-Romeiser spectrum were obtained at wind speeds below $14 \mathrm{~m} \mathrm{~s}^{-1}$. At higher wind speeds, these model spectra are used formally but without any experimental confirmation.

Figure $9 \mathrm{~b}$ shows the coefficient of mass exchange via the wind speed. The difference in the values calculated within the different models is quite significant, but it does not exceed experimental errors. The best agreement is achieved with calculations using the Hwang-Romeiser spectrum. The dependence of $C_{\rho_{10 \mathrm{~N}}}\left(U_{10 \mathrm{~N}}\right)$ is slower than $C_{\mathrm{D}_{10 \mathrm{~N}}}\left(U_{10 \mathrm{~N}}\right)$. This is easy to explain, using the definitions in Eq. (38), from which we find that

$C_{\rho_{10 \mathrm{~N}}}=\frac{\rho_{*} u_{*}}{\Delta \rho_{10 \mathrm{~N}} U_{10 \mathrm{~N}}}=\frac{\rho_{*}}{\Delta \rho_{10}} \sqrt{C_{\mathrm{D}_{10 \mathrm{~N}}}}$.

The calculations within the framework of CM1 showed that the profile of the average density is practically independent of the waves at the water surface, which means that one can use the following for $\Delta \rho_{10}(\eta)$ expression for the case of smooth surface:

$\Delta \rho_{10}=\frac{\rho_{*}}{\kappa P r_{\mathrm{t}}} \ln \frac{\eta}{z_{\rho 0}}$

and the roughness parameter for the density $z_{\rho 0}=\frac{\alpha \nu}{u_{\star}}$, where $\alpha=0.177$, according to Liu et al. (1979).

As a result, we have for the mass exchange coefficient

$C_{\rho_{10 \mathrm{~N}}}=\sqrt{C_{\mathrm{D}_{10 \mathrm{~N}}}} \frac{\kappa P r_{\mathrm{t}}}{\ln \frac{H_{10} u_{*}}{\alpha \nu}}=\sqrt{C_{\mathrm{D}_{10 \mathrm{~N}}}} \frac{\kappa P r_{\mathrm{t}}}{\ln \frac{H_{10} U_{10} \sqrt{C_{\mathrm{D}_{10 \mathrm{~N}}}}}{\alpha \nu}}$.

The numerator and denominator are the increasing functions of $U_{10 \mathrm{~N}}$, but the result of their balance is a slightly increasing function $C_{\rho_{10 \mathrm{~N}}}\left(U_{10 \mathrm{~N}}\right)$. 


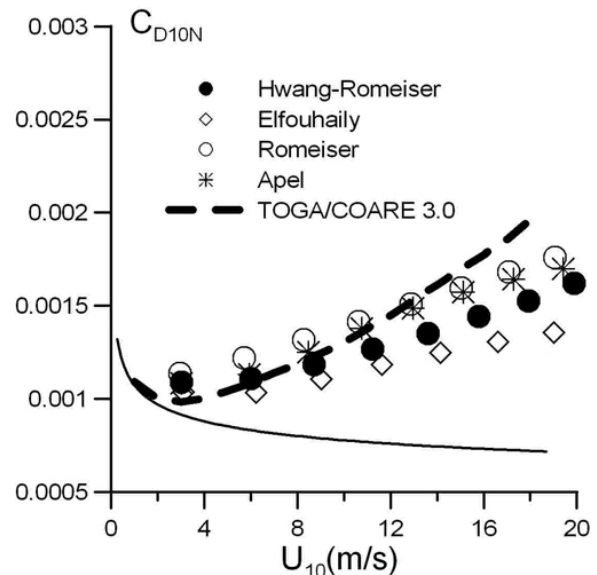

(a)

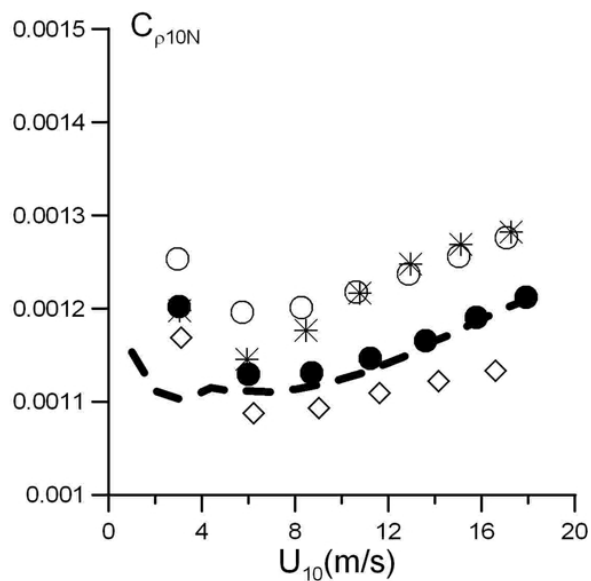

(b)

Fig. 9. Drag coefficient (a) and mass exchange coefficient (b) as functions of wind speed for different spectra. Thin solid curve corresponds to the flow above the smooth surface.

Table 1. Relative errors of coefficients $C_{D_{10 N}}$ and $C_{\rho_{10 \mathrm{~N}}}$, calculated within the framework of the model with different empirical wave spectra.

\begin{tabular}{lrrrr}
\hline & $\begin{array}{r}\text { Elfouhaily spectrum, } \\
\text { formulas (A1)-(A3) }\end{array}$ & $\begin{array}{r}\text { Apel spectrum, } \\
\text { formulas (A4)-(A7) }\end{array}$ & $\begin{array}{r}\text { Romeiser spectrum, } \\
\text { formulas (A8)-(A13) }\end{array}$ & $\begin{array}{r}\text { Hwang-Romeiser spectrum, } \\
\text { formulas (A14)-(A18) }\end{array}$ \\
\hline $\begin{array}{l}\varepsilon_{\mathrm{D}}, \\
U_{10}<18 \mathrm{~m} \mathrm{~s}^{-1}\end{array}$ & 0.27 & 0.10 & 0.11 & 0.12 \\
$\varepsilon_{\rho}$, & 0.25 & 0.07 & 0.08 & 0.03 \\
$U_{10}<18 \mathrm{~m} \mathrm{~s}^{-1}$ & 0.17 & 0.06 & 0.10 & 0.11 \\
$\varepsilon_{\mathrm{D}}$, & 0.04 & 0.07 & 0.09 & 0.04 \\
$U_{10}<14 \mathrm{~m} \mathrm{~s}^{-1}$ & & & & \\
$\varepsilon_{\rho}$, & & & \\
$U_{10}<14 \mathrm{~m} \mathrm{~s}^{-1}$ & & & \\
\hline
\end{tabular}

\section{Conclusions}

The question regarding the influence of the surface waves on air-sea momentum and mass exchange is one of the most important problems in theory of surface waves and MABL and its applications. A role of surface waves in formation of a sea surface drag is well established. In particular, it has been found that a drag coefficient is an increasing function of wind speed due to the wave spectrum expansion. To date, large amounts of experimental data on aerodynamic sea surface drag have been accumulated (Fairall et al., 2003; Brut et al., 2005; Zeng et al., 1998) that can be used for verification of the theoretical models of MABL above a wavy water surface. Dependence of mass exchange coefficient $C_{\rho}$ on wind speed is much less explored. The experiments (Fairall et al., 2003; Ocampo-Torres et al., 1994; Brut et al., 2005; Drennan et al., 2007) show that this dependence is weaker than for $C_{\mathrm{D}}$; however, statistically significant weak growth of heat and moisture exchange coefficients with wind speed has been proved (Fairall et al., 2003; Ocampo-Torres et al., 1994; Brut et al., 2005).
In the present work we suggest a self-consistent model of stratified turbulent boundary layer over waved water surface. The model is based on the system of Reynolds-averaged Navier-Stokes equations in the basic formulation with the first-order closure hypothesis, where coefficients of turbulent transport were verified experimentally. Comparisons with the experimental data enabled us to choose the eddy viscosity and heat conductivity coefficients scaled by the total tangential stress in the boundary layer. Wind-wave momentum and mass exchange within the model is considered in the quasilinear approximation. This approach, when the linear approximation is prescribed for the wave-induced disturbances and nonlinear effects are concerned only with the mean flow, is often used in plasma physics. For the wind-wave interaction with homogeneous MABL, it was applied by Janssen, 1991; Fabricant, 1976; Jenkins, 1992; and Reutov and Troitskaya, 1996. Wave momentum and mass fluxes in this approach are calculated from the solution of the boundary problem for coupled systems of the wave-induced disturbances and mean fields.

As nonlinear corrections to mean wind speed and density profiles are determined by a wave number-frequency 
spectrum, it is an important element of the model. In the present model for calculation of drag and mass exchange coefficients, we used several model spectra, describing wave parameters within the interval from millimeters to some hundred meters wave lengths (Elfouhaily et al., 1997; Apel, 1994; Romeiser et al., 1997; Hwang et al., 1996, 2001a, b; Hwang, 1997, 2005; Hwang and Wang, 2004). It was shown that the main contribution to the resistance of the surface is made by the waves with wave lengths from meters to centimeters. The best agreement of theoretically calculated drag coefficient with TOGA/COARE (Fairall et al., 2003) data is achieved for the Hwang spectrum (Hwang, 2005), where the high-frequency part is completed by the Romeiser spectrum (Romeiser et al., 1997). At the moment it is the only model of the wind-wave spectrum where the short wave part (from $6 \mathrm{~m}$ to $4 \mathrm{~cm}$ ) is based on field data. Other spectra use data from laboratory experiments, which can significantly differ from field data (see, e.g., Hwang et al., 1996). The additional advantage of this spectrum is agreement of a calculated mean square slope with data from Cox and Munk (1954). The data are in good agreement with the experiment at wind speeds up to about $10 \mathrm{~m} \mathrm{~s}^{-1}$; at higher winds the model underestimates $C_{\mathrm{D}_{10 \mathrm{~N}}}$ by about $10 \%$. A possible explanation is the incorrect extension of the short wave spectra to the case of strong winds.

Calculations within the model showed that the coefficient of mass exchange $C_{\rho_{10 \mathrm{~N}}}\left(U_{10 \mathrm{~N}}\right)$ increased with the wind speed, although the growth is slower than for the momentum exchange coefficient $C_{\mathrm{D}_{10 \mathrm{~N}}}\left(U_{10 \mathrm{~N}}\right)$. According to its definition, $C_{\rho_{10 \mathrm{~N}}}\left(U_{10 \mathrm{~N}}\right)$ is a dimensionless number that measures the ratio of mass transferred from the air to water to wind speed and density differences between air and water. Note that according to the model the density profile in MABL is practically independent of waves. Also, the mass exchange coefficient is determined by balance between reduction of mass transfer in viscous sublayer due to decrease of the density roughness parameter and increase of the momentum exchange coefficient with wind speed. There is only an indirect effect of wave disturbances on the mass transfer in this case, which is associated with an increase in the coefficient of momentum exchange with increasing wind speed - which in turn is due to the transfer pulse waves.

\section{Appendix A}

\section{Model spectra of short wind waves}

\section{A1 Elfouhaily spectrum}

Surface elevation spectrum can be represented as a sum of two items $S_{\text {elf }}=\left(B_{1}+B_{\mathrm{h}}\right) D_{\text {elf }}(\phi) / k^{3}$, long wave and short wave; for each component, parameterization of saturation spectra $B_{1}$ and $B_{\mathrm{h}}$ is suggested. For a long wave part of the saturation spectrum, the Donelan-Pierson formula
(Donelan and Pierson, 1987) is suggested (see Elfouhaily et al., 1997):

$$
\begin{aligned}
& B_{1}=\frac{0.006}{2} \Omega^{0.55}\left(\frac{k}{k_{\mathrm{p}}}\right)^{1 / 2}
\end{aligned}
$$

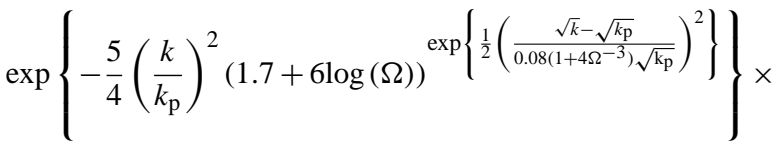

$$
\begin{aligned}
& \times \exp \left[-\frac{\Omega}{\sqrt{10}}\left(\sqrt{\frac{k}{k_{\mathrm{p}}}}-1\right)\right] \text {, }
\end{aligned}
$$

where $\Omega=\frac{U_{10} \sqrt{k_{\mathrm{p}}}}{\sqrt{g}}$ is wave age. For a short wave part of the saturation spectrum, Elfouhaily et al. (1997) use the following formula:

$B_{\mathrm{h}}=\frac{10^{-2}}{2}\left(1+3 \ln \frac{u_{*}}{c}\right) \frac{u_{*}}{c} e^{-\frac{1}{4}\left(\frac{k}{k_{\mathrm{m}}}-1\right)^{2}} ; k_{\mathrm{m}}=\frac{2 g}{\left(23 \mathrm{cms}^{-1}\right)^{2}}$.

The following approximation (see Elfouhaily et al., 1997) is used as an angle distribution of elevation spectrum:

$$
\begin{aligned}
& D(\phi)=\frac{1}{\pi}(1+\delta \cos (2 \phi)), \text { where } \\
& \delta=\operatorname{atan}\left[\frac{\log (2.0)}{4}+4\left(\frac{c}{c_{\mathrm{p}}}\right)^{2.5}+0.13 \frac{u_{*}}{23}\left(\frac{c}{23}\right)^{-2.5}\right] .
\end{aligned}
$$

\section{A2 Apel spectrum}

This spectrum can be represented as a product of DonelanPierson spectrum (Donelan and Pierson, 1987):

$$
\begin{aligned}
& S_{\text {apel }}=S_{1} W D_{\text {apel }}(\phi), \\
& S_{1}=\frac{0.00195}{k^{3}} \exp \left(-\left(\frac{k_{\mathrm{p}}}{k}\right)^{2}\right) 1.7^{r}, \\
& \text { Гдеr }=\exp \left(-\frac{1}{0.32}\left(\sqrt{\frac{k}{k_{\mathrm{p}}}}-1\right)^{2}\right),
\end{aligned}
$$

multiplied by the sum of a low frequency filter with a cutoff wave number $k_{0}=1 \mathrm{~cm}^{-1}$ and a band-pass filter in the vicinity of a wave number $k_{1}=4 \mathrm{~cm}^{-1}$.

$$
\begin{aligned}
& W=\left[\frac{1.0}{\left(k / k_{0}\right)^{2}+1}+\frac{a k}{\cosh \left(\frac{k-k_{1}}{k_{2}}\right)} 10\left(-4.95+3.45 *\left(1-e^{-\frac{U_{10}}{U_{0}}}\right)\right)\right] \\
& \exp \left(-\left(\frac{k}{k_{3}}\right)^{2}\right) .
\end{aligned}
$$

Here, $k_{2}=4.5 \mathrm{~cm}^{-1}, k_{3}=62.83 \mathrm{~cm}^{-1}$, and $a=80 \mathrm{~cm}$, $U_{0}=4.7 \mathrm{~m} \mathrm{~s}^{-1}$.

Angle distribution of wave spectral density in the Apel spectrum (Apel, 1994) is a Gaussian function:

$$
D_{\text {apel }}(\phi)=\exp \left(-4 \phi^{2}\left(0.14+5 \frac{k}{k_{\mathrm{p}}}\right)^{-1.3}\right) \text {. }
$$


Table A1. Polynomial coefficients for $A_{0}$ and $a_{0}$ in Eq. (A16).

\begin{tabular}{rrrrrrrrrrr}
\hline$C_{0}$ & $C_{1}$ & $C_{2}$ & $C_{3}$ & $C_{4}$ & $C_{5}$ & $C_{6}$ & $C_{7}$ & $C_{8}$ & $C_{9}$ & $C_{10}$ \\
-5.62 & -1.03 & 2.20 & 6.76 & 8.47 & 6.05 & 2.64 & 0.717 & 0.117 & 0.0106 & 0.000403 \\
\hline$c_{0}$ & $c_{1}$ & $c_{2}$ & $c_{3}$ & $c_{4}$ & $c_{5}$ & $c_{6}$ & $c_{7}$ & $c_{8}$ & $c_{9}$ & $c_{10}$ \\
1.39 & 0.736 & 0.516 & 1.07 & 1.48 & 1.20 & 0.585 & 0.174 & 0.0308 & 0.00296 & 0.000119 \\
\hline
\end{tabular}

\section{A3 Modified Apel spectrum, suggested by Romeiser et al. (1997)}

This spectrum is a modified Apel spectrum (Apel, 1994) allowing for data on the scattering cross-section of radio waves with wave lengths from dm to $\mathrm{mm}$. Spectral density of surface elevation is given by the following expression:

$S_{\mathrm{r}}=P_{L}\left(\frac{U_{10}}{u_{k}}\right)^{\beta_{k}} W_{\mathrm{h}}(k) D_{\mathrm{r}}(\phi)$,

where

$$
\begin{aligned}
P_{L} & =\frac{0.00195}{k^{3}} \exp \left(-\left(\frac{k_{\mathrm{p}}}{k}\right)^{2}+0.53\right. \\
& \exp \left(-\frac{1}{0.32}\left(\sqrt{\left.\frac{k}{k_{\mathrm{p}}}-1\right)}\right)^{2}\right), \\
\beta_{k} & =\left(1-\exp \left(-\frac{k^{2}}{k_{1}^{2}}\right)\right) \exp \left(-\frac{k^{2}}{k_{2}^{2}}\right) \\
& \left.\left.+\left(1-\exp \left(-\frac{k^{2}}{k_{3}^{2}}\right)\right)^{\exp \left(-k^{2}\right.}\right)^{2}\right), \\
W_{\mathrm{h}}(k) & =\frac{\left(1+\left(\frac{k}{k_{6}}\right)^{7.2}\right)^{0.5}}{\left(1+\left(\frac{k}{k_{7}}\right)^{2.2}\right)\left(1+\left(\frac{k}{k_{8}}\right)^{3.2}\right)^{2}} \\
& \exp \left(-\left(\frac{k}{k_{9}}\right)^{2}\right)^{2}
\end{aligned}
$$

Numeric parameters, included in Eqs. (A10)-(A11), are the following:

$k_{1}=1.83 \mathrm{~cm}^{-1}, \quad k_{2}=33.33 \mathrm{~cm}^{-1}, \quad k_{3}=0.33 \mathrm{~cm}^{-1}$, $k_{4}=1.4 \mathrm{~cm}^{-1}, k_{5}=2.2 \mathrm{~cm}^{-1}, k_{6}=2.8 \mathrm{~cm}^{-1}$,

$k_{7}=0.75 \mathrm{~cm}^{-1}, \quad k_{8}=13 \mathrm{~cm}^{-1}, \quad k_{9}=88.85 \mathrm{~cm}^{-1}$, $u_{k}=1 \mathrm{~m} \mathrm{~s}^{-1}$.

Angle distribution of spectral density of surface elevation is formulated as

$D_{\mathrm{r}}(\phi)=\exp \left(-\phi^{2} d_{\phi}\right)$ where

$$
\begin{aligned}
d_{\phi} & =0.14+0.5\left(1-\exp \left(-\frac{k U_{10}}{\omega_{n}}\right)\right) \\
& +5 \exp \left[2.5-2.6 \log \left(\frac{U_{10}}{u_{k}}\right)-1.3 \log \left(\frac{k}{k_{n}}\right)\right]
\end{aligned}
$$

in which $\omega_{n}=400 \mathrm{~s}^{-1}, k_{n}=10^{-2} \mathrm{~cm}^{-1}$.

\section{A4 Hwang spectrum}

In the papers by Hwang et al. (1996, 2001a, b), Hwang (1997, 2005) and Hwang and Wang (2004), the following expression for the surface elevation spectrum is suggested:

$S_{\mathrm{h}}=\frac{A_{0}(k)}{k^{3}}\left(\frac{u_{*}}{c}\right)^{a_{0}(k)} D_{\mathrm{h}}(\phi)$,

where angle distribution is

$D_{\mathrm{h}}(\phi)=\frac{1}{\pi}\left[1+\sum_{n=1}^{N} A_{n}(k) \cos (2 n \phi)\right]$.

Coefficients $C_{n}$ are given in Hwang et al. (2001b) as polynomials of $k$; for $k>2 k_{\mathrm{p}}$ the angle distribution becomes bimodal.

Note that this spectrum is special as it is obtained from the approximation of field observations data, including $\mathrm{cm}$ wave lengths. This is not the case for other spectra (Elfouhaily et al., 1997; Apel, 1994; Romeiser et al., 1997), that are based on the data of laboratory experiments. At the same time, according to Hwang et al. (1996), there are significant discrepancies between field and laboratory data, in particular, dependencies of saturated spectral densities on wind speed are different for $\mathrm{cm}$ and $\mathrm{dm}$ wave lengths. For instance, the growth of a high-frequency part of a spectrum with wind speed is slower in field conditions compared to laboratory conditions (Hwang et al., 1996, 2001a, b; Hwang, 1997, 2005; Hwang and Wang, 2004). Also, Hwang spectrum has a bimodal angle distribution of spectral density, unlike to Elfouhaily et al. (1997), Apel (1994) and Romeiser et al. (1997).

According to Hwang (2005), for $k<1 \mathrm{~m}^{-1}$ coefficients $A_{0}=0.0526$ and $a_{0}=1$ in formula (A14); for $1 \mathrm{~m}^{-1}<k<$ $3.1623 \mathrm{~cm}^{-1}$ they are given in tables. For convenience of calculations, we suggest approximations of these curves by 
polynomials of the 10th order:

$$
\begin{gathered}
A_{0}=\exp \left[\sum_{n=0}^{10} C_{n} \log \left(\frac{k}{k_{0}}\right)^{n}\right] ; a_{0}=\sum_{n=0}^{10} c_{n} \log \left(\frac{k}{k_{0}}\right)^{n}, \\
k_{0}=3.1623 \mathrm{~cm}^{-1}
\end{gathered}
$$

being in very good agreement with the curves in Hwang (2005). Coefficients of the polynomials are given in Table A1. For $k>3.1623 \mathrm{~cm}^{-1}$ we completed the spectrum of Hwang (2005) by spectrum of Romeiser et al. (1997), described earlier.

Note that polynomial approximations in angle distribution (coefficients $A_{n}$ in Eq. A15) are justified only for the long wave part of the spectrum, for $k / k_{\mathrm{p}}<9$. In the present work we used the following model of angle spectrum distribution:

$D_{\mathrm{h}}(\phi)=\left\{\begin{array}{l}\frac{1}{\pi}\left[1+\sum_{n=1}^{N} A_{n}(k) \cos (2 n \phi)\right] ; k<9 k_{\mathrm{p}}, \\ \frac{1}{\pi}\left[1+\sum_{n=1}^{N} A_{n}\left(9 k_{\mathrm{p}}\right) \cos (2 n \phi)\right] ; 9 k_{\mathrm{p}}<k<1 m^{-1} .\end{array}\right.$

For $k>1 \mathrm{~m}^{-1}$ we used angle distribution (A12), suggested in Romeiser et al. (1997), with a necessary normalization:

$D_{\mathrm{hl}}(\phi)=\exp \left(-\phi^{2} d_{\phi}\right) \sqrt{\frac{\pi}{d_{\phi}}}$.

The disadvantage of this representation is a sudden change of angle spectrum distribution for $k=1 \mathrm{~m}^{-1}$. At the same time, in the model developed here the spectrum is integrated by wave numbers, making the model insensitive to such changes in integrands.

Acknowledgements. This work was supported by a grant from the Government of the Russian Federation under contract no. 11.G34.31.0048; the European Research Council Advanced Grant, FP7-IDEAS, 227915; RFBF grants 13-05-00865-a, 1305-97068, 13-05-12093, 12-05-01064, 12-05-31435 mol_a, 12-05-33070 mol_a_ved, Federal Targeted Program "Scientific and Education Personnel of Innovative Russia" for 2009-2013 GK No. 14.132.21.1384, GK No. 16.740.11.0673.

Edited by: I. Esau

Reviewed by: two anonymous referees

\section{References}

Apel, J. R.: An improved model of the ocean surface wave vector spectrum and its effects on radar backscatter, J. Geophys. Res., 99, 16269-16291, 1994.

Brut, A., Butet, A., Durand, P., Caniaux, G., and Planton, S.: Airsea exchanges in the equatorial area from the EQUALANT99 dataset: Bulk parametrizations of turbulent fluxes corrected for airflow distortion, Q. J. R. Meteorol. Soc., 131, 2497-2538, 2005.
Cox, C. S. and Munk W. H.: Statistics of the sea surface derived from sun glitter, J. Marine Res., 13, 198-227, 1954.

Donelan, M. A. and Pierson, W. J.: Radar scattering and equilibrium ranges in wind-generated waves - with application to scatterometry, J. Geophys. Res. Oceans, 92, 4971-5029, 1987.

Drennan, W. M., Zhang, J., French, J. R., McCormick, C., and Black, P. G.: Turbulent fluxes in the hurricane boundary layer, Part II: Latent heat flux, J. Atmos. Sci., 64, 1103-1115, 2007.

Druzhinin, O. A., Troitskaya, Y. I., and Zilitinkevich, S. S.: Direct numerical simulation of a turbulent wind over a wavy water surface, J. Geophys. Res., 117, C00J05, doi:10.1029/2011JC007789, 2012.

Elfouhaily, T. B., Chapron, B., Katsaros, K. B., and Vandemark, D. J.: A unified directional spectrum for long and short wind-driven waves, J. Geophys. Res., 107, 15781-15796, 1997.

Fabricant, A. L.: Quasilinear theory of wind waves generation, Izvestiya, Atmos. Ocean. Phys., 12, 858-862, 1976.

Fairall, C. W., Bradley, E. F., Hare, J. E., Grachev, A. A., and Edson, J. B.: Bulk parameterization of air-sea fluxes: updates and verification for the COARE algorithm, J. Climate, 16, 571-591, 2003.

Haberman, R.: Wave-induced distortions of a slightly stratified shear flow: a nonlinear critical-layer effect, J. Fluid Mech., 58, 727-736, 1973.

Hwang, P. A.: A study of the wavenumber spectra of short water waves in the ocean, Part 2: spectral model and mean square slope, J. Atmos. Ocean. Technol., 14, 1174-1186, 1997.

Hwang, P. A.: Wave number spectrum and mean square slope of intermediate-scale ocean surface waves, J. Geophys. Res., 110, C10029, doi:10.1029/2005JC003002, 2005.

Hwang, P. A. and Wang, D. W.: An empirical investigation of source term balance of small scale surface waves, Geophys. Res. Lett., 31, L15301, doi:10.1029/2004GL020080, 2004.

Hwang, P. A., Atakturk, S., Sletten, M. A., and Trizna, D. B.: A study of the wavenumber spectra of short water waves in the ocean, J. Phys. Oceanogr., 26, 1266-1285, 1996.

Hwang, P. A., Wang, D. W., Walsh, E. J., Krabill, W. B., and Swift, R. N.: Airborne measurements of the wavenumber spectra of ocean surface waves, Part 1: spectral slope and dimensionless spectral coefficient, J. Phys. Oceanogr., 30, 2753-2767, 2001a.

Hwang, P. A., Wang, D. W., Walsh, E. J., Krabill, W. B., and Swift R. N.: Airborne measurements of the wavenumber spectra of ocean surface waves. Part 2: directional distribution, J. Phys. Oceanogr., 30, 2768-2787, 2001b.

Janssen, P. A. E. M.: Quasi-linear theory of wind wave generation applied to wave forecasting, J. Phys. Oceanogr., 21, 1631-1642, 1991.

Jenkins, A. D.: Quasi-linear eddy-viscosity model for the flux of energy and momentum to wind waves using conservation-law equations in a curvilinear coordinate system, J. Phys. Oceanogr., 22, 843-858, 1992.

Liu, W. T., Katsaros, K. B., and Businger, J. A.: Bulk parameterization of air-sea exchanges of heat and water vapor including the molecular constraints at the interface, J. Atmos. Sci., 36, 17221735, 1979.

Makin, V. K. and Kudryavtsev, V. N.: Coupled sea surfaceatmosphere model, Part 1. Wind over waves coupling, J. Geophys. Res., 104, 7613-7623, 1999. 
Makin, V. K. and Mastenbroek, C.: Impact of waves on air-sea exchange of sensible heat and momentum, Bound.-Lay. Meteorol., 79, 279-300, 1996.

Makin, V. K., Kudryavtsev, V. N., and Mastenbroek, C.: Drag of the sea surface, Bound.-Lay. Meteorol., 73, 159-182, 1995.

Maslowe, S. A.: The generation of clear air turbulence by nonlinear waves, Stud. Appl. Math., 51, 1-16, 1972.

Monin, A. S. and Yaglom, A. M.: Statistical fluid dynamics, V.1. Gidrometeoizdat, St. Petersburg, 1992.

Ocampo-Torres, F. J., Donelan M. A., Merzi N., and Jia, F.: Laboratory measurements of mass transfer of carbon dioxide and water vapour for smooth and rough flow conditions, Tellus Ser. B, 46, 16-32, 1994.

Reutov, V. P.: Nonlinear growth rate of wind water waves and their excitation near the stability threshold, Radiophys. Quantum Electron., 38, 133-136, 1995.

Reutov, V. P. and Troitskaya, Yu. I.: Nonlinear effects due to water wave interactions with a turbulent wind, Izvestyia, Atmos. Ocean. Phys., 31, 792-801, 1996.

Romeiser, R., Alpers, W., and Wismann, V.: An improved composite surface model for the radar backscattering cross section of the ocean surface, J. Geophys. Res., 102, 25237-25250, 1997.

Smol'yakov, A. V.: Quadrupole radiation spectrum of plane turbulent boundary layer, Sov. Phys. Acoust., 19, 271-276, 1973.
Troitskaya, Yu. I.: Viscous diffusion nonlinear critical layer in a stratified shear flow, J. Fluid Mech., 233, 25-48, 1991.

Troitskaya, Yu. I. and Rybushkina, G. V.: Quasi-linear model of interaction of surface waves with strong and hurricane winds, Izvestyia, Atmos. Ocean. Phys., 44, 621-645, 2008.

Troitskaya, Yu. I., Sergeev, D. A., Ermakova, O. S., and Balandina, G. N.: Statistical parameters of the air turbulent boundary layer over steep water waves measured by the DPIV technique, J. Phys. Oceanogr., 41, 1421-1454, 2011.

Troitskaya, Yu. I., Sergeev, D. A., Kandaurov, A. A., Baidakov, G. A, Vdovin, M. A., and Kazakov, V. I.: Laboratory and theoretical modeling of air-sea momentum transfer under severe wind conditions, J. Geophys. Res., 117, C00J21, doi:10.1029/2011JC007778, 2012.

Troitskaya, Yu. I., Ezhova, E. V., and Zilitinkevich, S. S.: Momentum and buoyancy transfer in atmospheric turbulent boundary layer over wavy water surface - Part 1: Harmonic wave, Nonlin. Processes Geophys., 20, 825-839, doi:10.5194/npg-20-8252013, 2013.

Zeng, X., Zhao, M., and Dickinson, R. E.: Comparison of bulk aerodynamic algorithms for the computation of sea surface fluxes using the TOGA COARE and TAO data, J. Climate, 11, 26282644, 1998. 